\title{
The influence of quark energy loss on extracting nuclear sea quark distribution from nuclear Drell-Yan experimental data
}

\author{
Duan Chun-Gui ${ }^{1,2,4}$, Liu Na ${ }^{1,3}$
}

\begin{abstract}
1.Department of Physics, Hebei Normal University, Shijiazhuang 050016, China
2.Hebei Advanced Thin Films Laboratory, shijiazhuang 050016, China

3.College of Mathematics and Physics, Shijiazhuang University of Economics, Shijiazhuang 050031, China

4.CCAST(World Laboratory), P.O.Box8730,Beijing 100080,China
\end{abstract}

\begin{abstract}
By means of two typical kinds of quark energy loss parametrization and the nuclear parton distributions determined only with lepton-nuclear deep inelastic scattering experimental data, a leading order analysis are performed on the proton-induced Drell-Yan differential cross section ratios of tungsten versus deuterium as a function of the quark momentum fraction in the beam proton and target nuclei. It is found that the theoretical results with quark energy loss are in good agreement with the experimental data. The quark energy loss effect produce approximately $3 \%$ to $11 \%$ suppression on the Drell-Yan differential cross section ratios $R_{W / D}$ in the range $0.05 \leq x_{2} \leq 0.3$. The application of nuclear Drell-Yan data with heavy targets is remarkably subject to difficulty in the constraints of the nuclear sea-quark distribution.
\end{abstract}

PACS: 12.38.-t;13.85.Qk;24.85.+p;25.40.-h

Keywords: Drell-Yan, Energy loss, nuclear sea quark distribution

After the discovery of the EMC effect ${ }^{[1]}$, it is found that the parton distributions are modified in the nuclear environment. The origin of the nuclear effects is still under debate in theory, and it is considered that different mechanisms are responsible for the effects in the different regions of parton momentum fraction. Up to now, almost all of the data on nuclear dependence is from charged lepton deep inelastic scattering experiments, which are sensitive only to the charge-weighted sum of all quark and antiquark distributions. From the charged lepton deep inelastic scattering off nuclei,

* E-mail:duancg@mail.hebtu.edu.cn 
the nuclear valence quark distributions are relatively well determined in the medium and large Bjorken $x$ regions. However, the charged lepton deep inelastic scattering experiment would not be sensitive to the nuclear sea quark distributions. In order to pin down the nuclear antiquark distributions, it is desirable that the nuclear Drell-Yan reaction is an ideal complementary tool.

The production of large-mass lepton pairs from hadron-hadron inelastic collisions was first studied by Drell and Yan, which is so-called Drell-Yan process ${ }^{[2]}$. According to the parton model, the process is induced by the annihilation of a quark-antiquark pair into a virtual photon which subsequently decays into a lepton pair. The nuclear Drell-Yan process of proton-nucleus collisions therefore is closely related to the quark distribution functions in nuclei. It is further natural to expect that the nuclear DrellYan reaction is a complementary tool to probe the structure of hadron and nuclei. The nuclear Drell-Yan experimental data can be used to research the anti-quark distributions in hadron and nuclei. However, it is indicated that the projectile rarely retains a major fraction of its momentum in traversing the nucleus in high energy inelastic proton-nucleus scattering. The quark and gluon in the induced proton can loss a finite fraction of its energy due to the multiple collisions in the nuclear target. In this respect, the initial-state interactions should be important in nuclear Drell-Yan process since the dimuon in the final state does not interact strongly with the partons in the nuclei. The quark energy loss effect in nuclear Drell-Yan process is another nuclear effect apart from the nuclear effects on the parton distribution as in deep inelastic scattering.

It is well known that the precise nuclear parton distributions are very important to explain high energy nuclear scattering phenomena. As for the strong necessities, the global analysis of nuclear parton distribution functions have been proposed in the recent years. For example, the global analysis of nuclear parton distribution functions are given by EKRS(Eskola, Kolhinen, Ruuskanen and Salgado) ${ }^{[3]}$, HKN(Hirai, Kumano and Nagai) ${ }^{[4]}$ and HKM (Hirai, Kumano and Miyama) ${ }^{[5]}$. It is noticeable that 
HKN employed Fermilab E772 ${ }^{[6]}$ and E866 ${ }^{[7]}$ nuclear Drell-Yan reaction data, EKRS included E772 experimental data, and HKM proposed the nuclear parton distributions which were determined by means of the existing experimental data on nuclear structure functions without including the proton-nucleus Drell-Yan process.

Because of the quark energy loss effect in nuclear Drell-Yan reaction, there is the kind of nuclear effect on the differential cross section ratios as a function of the quark momentum fraction in the beam proton and target nuclei. In previous works ${ }^{[8-11]}$, the Fermilab E866 ${ }^{[7]}$ nuclear Drell-Yan reaction data were used to investigate quark energy loss effect. But, the impact of quark energy loss can be canceled partly out in the proton-induced Drell-Yan cross section ratios for Fe to Be and $\mathrm{W}$ to Be. In this paper, by combining two typical kinds of quark energy loss parametrization with the nuclear parton distributions determined only with lepton-nuclear deep inelastic scattering experimental data, a leading order analysis are performed on the differential cross section ratios of tungsten versus deuterium as a function of the quark momentum fraction in the beam proton and target nuclei for the proton-induced Drell-Yan process. The influence of quark energy loss is elucidated on extracting nuclear sea-quark distribution.

In the Drell-Yan process $^{[2]}$, the leading-order contribution is quark-antiquark annihilation into a lepton pair. The annihilation cross section can be obtained from the $q \bar{q} \rightarrow l^{+} l^{-}$cross section, which is

$$
\sigma\left[q \bar{q} \rightarrow l^{+} l^{-}\right]=\frac{4 \pi \alpha_{e m}^{2}}{9 M^{2}} e_{f}^{2}
$$

where $\alpha_{e m}$ is the fine-structure constant, $e_{f}$ is the charge of the quark and $M$ is the invariant mass of the lepton pair. The nuclear Drell-Yan differential cross section can be written as

$$
\frac{d^{2} \sigma}{d x_{1} d x_{2}}=\frac{4 \pi \alpha_{e m}^{2}}{9 s x_{1} x_{2}} \sum_{f} e_{f}^{2}\left[q_{f}^{p}\left(x_{1}, Q^{2}\right) \bar{q}_{f}^{A}\left(x_{2}, Q^{2}\right)+\bar{q}_{f}^{p}\left(x_{1}, Q^{2}\right) q_{f}^{A}\left(x_{2}, Q^{2}\right)\right]
$$


where $\sqrt{s}$ is the center of mass energy of the hadronic collision, $x_{1}\left(\right.$ resp. $\left.x_{2}\right)$ is the momentum fraction carried by the projectile (resp.target) parton, the sum is carried out over the quark flavor $f$, and $q_{f}^{p(A)}\left(x, Q^{2}\right)$ and $\bar{q}_{f}^{p(A)}\left(x, Q^{2}\right)$ are respectively the quark and anti-quark distributions in the proton (nucleon in the nucleus A).

In order to take into account of the energy loss of the fast quarks moving through the cold nuclei, we will introduce two typical kinds of quark energy loss expressions, which are based on the theoretical studies from Brodsky and Hoyer ${ }^{[12]}$, and Baier et.al. ${ }^{[13]}$. One can be rewritten as

$$
\Delta x_{1}=\alpha \frac{<L>_{A}}{E_{p}}
$$

where $\alpha$ indicates the incident quark energy loss per unit length in nuclear matter, $<L>_{A}$ is the average path length of the incident quark in the nucleus $\mathrm{A}, E_{p}$ is the energy of the incident proton. The average path length is employed by the conventional value, $<L>_{A}=3 / 4\left(1.2 A^{1 / 3)} \mathrm{fm}\right.$. In addition to the linear quark energy loss rate, another is rewritten as

$$
\Delta x_{1}=\beta \frac{<L>_{A}^{2}}{E_{p}}
$$

Obviously, the partonic energy loss is quadratic with the path length. With considering the quark energy loss in nuclei, the incident quark momentum fraction can be shifted from $x_{1}^{\prime}=x_{1}+\Delta x_{1}$ to $x_{1}$ at the point of fusion. Thus, the nuclear Drell-Yan differential cross section can be expressed as

$$
\frac{d^{2} \sigma}{d x_{1} d x_{2}}=\frac{4 \pi \alpha_{e m}^{2}}{9 s x_{1} x_{2}} \sum_{f} e_{f}^{2}\left[q_{f}^{p}\left(x_{1}^{\prime}, Q^{2}\right) \bar{q}_{f}^{A}\left(x_{2}, Q^{2}\right)+\bar{q}_{f}^{p}\left(x_{1}^{\prime}, Q^{2}\right) q_{f}^{A}\left(x_{2}, Q^{2}\right)\right]
$$

After the integral of the differential cross section above, the nuclear Drell-Yan production cross section is given by

$$
\frac{d \sigma}{d x_{1(2)}}=\int d x_{2(1)} \frac{d^{2} \sigma}{d x_{1} d x_{2}}
$$

The Fermilab Experiment772(E772) [6] reported the ratios of proton-induced 
nuclear Drell-Yan differential cross section for tungsten to deuterium target,

$$
R_{W / D}\left(x_{1(2)}\right)=\frac{d \sigma^{p-W}}{d x_{1(2)}} / \frac{d \sigma^{p-D}}{d x_{1(2)}} .
$$

Muon pairs were recorded in the range $4 G e V \leq M \leq 9 G e V$ and $M \geq 11 G e V$. The covered kinematical range was $0.1<x_{2}<0.3$. By combining the quark energy loss effect with HKM ${ }^{[5]}$ cubic type of nuclear parton distributions determined only from lepton-nuclear deep inelastic scattering experimental data, a global $\chi^{2}$ analysis is given to the experimental data on the ratios of nuclear Drell-Yan differential cross section $R_{W / D}\left(x_{1(2)}\right)$

As for the rations $R_{W / D}\left(x_{1}\right)$, the obtained $\chi^{2}$ per degrees of freedom is $\chi^{2} /$ d.o.f. $=$ 2.14 for the 31 data points without energy loss effect. It is shown that the calculated results without energy loss effect are in disagreement with the experimental data. With the fast quark energy loss, the $\chi^{2}$ per degrees of freedom is $\chi^{2} /$ d.o.f. $=0.84$ for the linear quark energy loss formula with $\alpha=1.28$, and the $\chi^{2}$ per degrees of freedom is given by $\chi^{2} /$ d.o.f. $=0.84$ for the quadratic quark energy loss expression with $\beta=0.2$. It is found that the results given by the linear quark energy loss are nearly the same as these from the quadratic quark energy loss. The nuclear Drell-Yan differential cross section ratios are shown in Fig. 1 as functions of $x_{1}$ for various interval of $M$, respectively. The solid curves are the ratios with only the nuclear effects on the parton distribution. The dotted curves correspond to the results by combining an linear quark energy loss with nuclear effects on structure function. It is apparent that the results with energy loss effect are in good agreement with the experimental data.

For the nuclear Drell-Yan differential cross section rations $R_{W / D}\left(x_{2}\right)$, the $\chi^{2}$ per degrees of freedom is given by $\chi^{2} /$ d.o.f. $=4.85$ for the 9 data points if the quark energy loss is not put in. There appears to be a significant disagreement between the theoretical results without energy loss effect and the experimental data. With considering the fast quark energy loss, the $\chi^{2}$ per degrees of freedom is $\chi^{2} /$ d.o.f. $=1.31$ for 
the linear quark energy loss formula with $\alpha=0.94$, and the $\chi^{2}$ per degrees of freedom is $\chi^{2} /$ d.o.f. $=1.32$ for the quadratic quark energy loss expression with $\beta=0.15$. It is shown that the results given by the linear quark energy loss are nearly identical with these from the quadratic quark energy loss. In Fig.2, the solid curve is the ratios with only the nuclear effects on the parton distributions, the dotted curve corresponds to the results with adding linear energy loss. Compared with the experimental data, our results with energy loss effect significantly agree with the experimental data. Meanwhile, It is noticeable that the values of the parameter $\alpha$ ( or $\beta$ ) are different by means of the global $\chi^{2}$ analysis to the ratios $R_{W / D}\left(x_{2}\right)$ and $R_{W / D}\left(x_{1}\right)$. The results may be originated from the experimental precision. If the experimental data are sufficiently precise, the values of the parameter $\alpha$ (or $\beta$ ) in the quark energy loss expression should be the same for fitting the ratios $R_{W / D}\left(x_{2}\right)$ and $R_{W / D}\left(x_{1}\right)$ from the nuclear Drell-Yan experiment.

In order to clarify the influence of quark energy loss on the Drell-Yan differential cross section ratios as a function of the quark momentum fraction in target nuclei, the ratios on $R_{W / D}\left(x_{2}\right)$ without quark energy loss to those with linear quark energy loss are calculated and tabulated in Table 1 . The similar results can be obtained for the quadratic quark energy loss. It is found that the quark energy loss effect make apparent influence on the Drell-Yan differential cross section ratios $R_{W / D}\left(x_{2}\right)$. The impact of quark energy loss on $R_{W / D}\left(x_{2}\right)$ become larger with the increase of momentum fraction of the target parton. The quark energy loss effect produce approximately $3 \%$ to $11 \%$ suppression on the Drell-Yan differential cross section ratios $R_{W / D}\left(x_{2}\right)$ in the ranges $0.05 \leq x_{2} \leq 0.3$. Therefore, the application of nuclear Drell-Yan data with heavy targets is remarkably subject to difficulty in the constraints of the nuclear antiquark distribution.

In summary, the quark energy loss effect in nuclear Drell-Yan process is another nuclear effect apart from the nuclear effects on the parton distribution as in deep 
TABLE 1: The ratios of $R_{W / D}\left(x_{2}\right)$ without quark energy loss to those with linear quark energy loss.

\begin{tabular}{ccccccc}
\hline$x_{2}$ & 0.05 & 0.10 & 0.15 & 0.20 & 0.25 & 0.30 \\
\hline the ratios of $R_{W / D}$ & 1.03 & 1.04 & 1.05 & 1.07 & 1.09 & 1.11 \\
\hline
\end{tabular}

inelastic scattering. From the global $\chi^{2}$ analysis on the ratios of nuclear Drell-Yan differential cross section $R_{W / D}\left(x_{1(2)}\right)$, it is found that the theoretical results with quark energy loss are in good agreement with the experimental data. The quark energy loss effect has significant impact on the Drell-Yan differential cross section ratio as a function of the quark momentum fraction in the beam proton and target nuclei. It is indicated that the influence of quark energy loss on the differential cross section ratios become greater with the increase of momentum fraction of the target parton. The application of nuclear Drell-Yan data with heavy targets is remarkably subject to difficulty in the constraint of the nuclear antiquark distribution. Therefore, we desire to operate precise measurements of the experimental study from the relatively low energy nuclear Drell-Yan process at J-PARC ${ }^{[14]}$ and Fermilab E906 ${ }^{[15]}$. These new experimental data on nuclear Drell-Yan reaction can shed light on the energy loss of fast quark propagating in a cold nuclei, and the impact of quark energy loss on determination nuclear sea-quark distribution .

Acknowledgement: This work is partially Supported by National Natural Science Foundation of China (10575028), Natural Science Foundation of Hebei Province (A2008000137).

\section{References}

[1] J.J.Aubert et al.,Phys.Lett.B,123(1983)275.

[2] S.Drell and T.M.Yan, Phys.Rev,Lett.,25(1970)316. 
[3] K.J.Eskola,V.J.Kolhinen and C.A.Salgado,Eur.Phys.J.C9(1999)61.

K.J.Eskola,V.J.Kolhinen and P.V.Ruuskanen,Nucl.Phys.B535(1998)351.

[4] M.Hirai,S.Kumano,T.H.Nagai, Phys.Rev. C70(2004)044905.

[5] M.Hirai,S.Kumano,M.Miyama, Phys.Rev.D64(2001)034003.

[6] D.M.Adle et al.,Phys.Rev.Lett.,64(1990)2479.

[7] M.A.Vasiliev,et.al.,Phys.Rev.Lett.,83(1999)2304.

[8] C.G.Duan, H.M. Wang and G.L. Li, Chin.Phys.Lett.19(2002)485.

[9] C.G. Duan et al., Eur. Phys. J. C29(2003)557, hep-ph/0405113.

[10] C.G. Duan et al., Eur. Phys. J. C39(2005)179, hep-ph/0601188.

[11] C.G. Duan et al., HEP \& NP, 30(2006)714.

[12] S.J.Brodsky and P.Hoyer,Phys.lett.,B298(1993)165.

[13] J.Badier.et.al.Nucl.Phys.B484(1997)265.

[14] M.Asakawa et.al.,KEK Report No 2000-11.

[15] D.Geesaman et al., Fermilab No.E906(1999). 

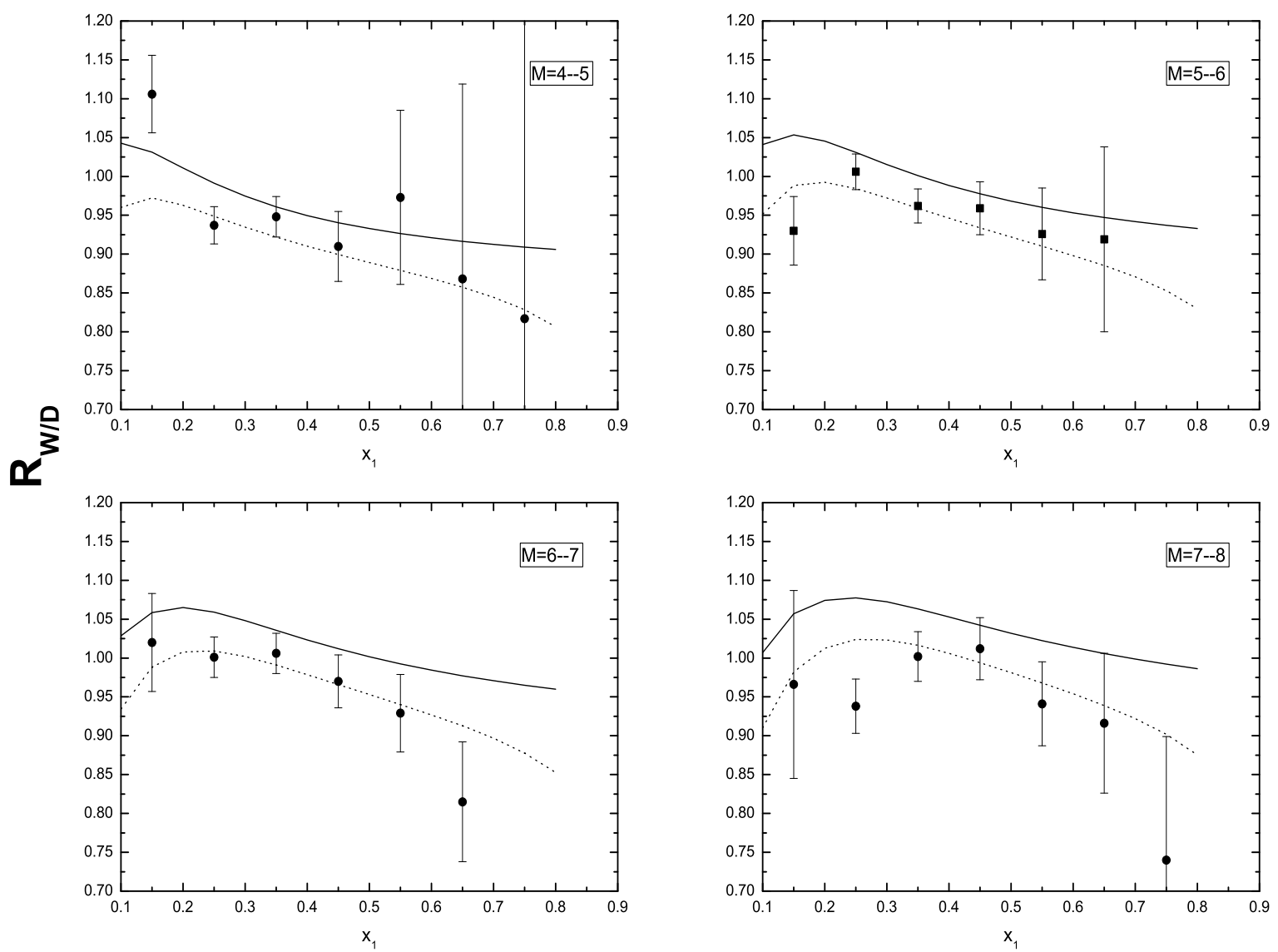

FIG. 1: The nuclear Drell-Yan cross section ratios $R_{W / D}\left(x_{1}\right)$ on for various intervals M. Solid curves correspond to nuclear effects on structure function. Dotted curves show the combination of linear quark energy loss effect with HKM cubic type of nuclear parton distributions. The experimental data are taken from E772 ${ }^{[6]}$. 


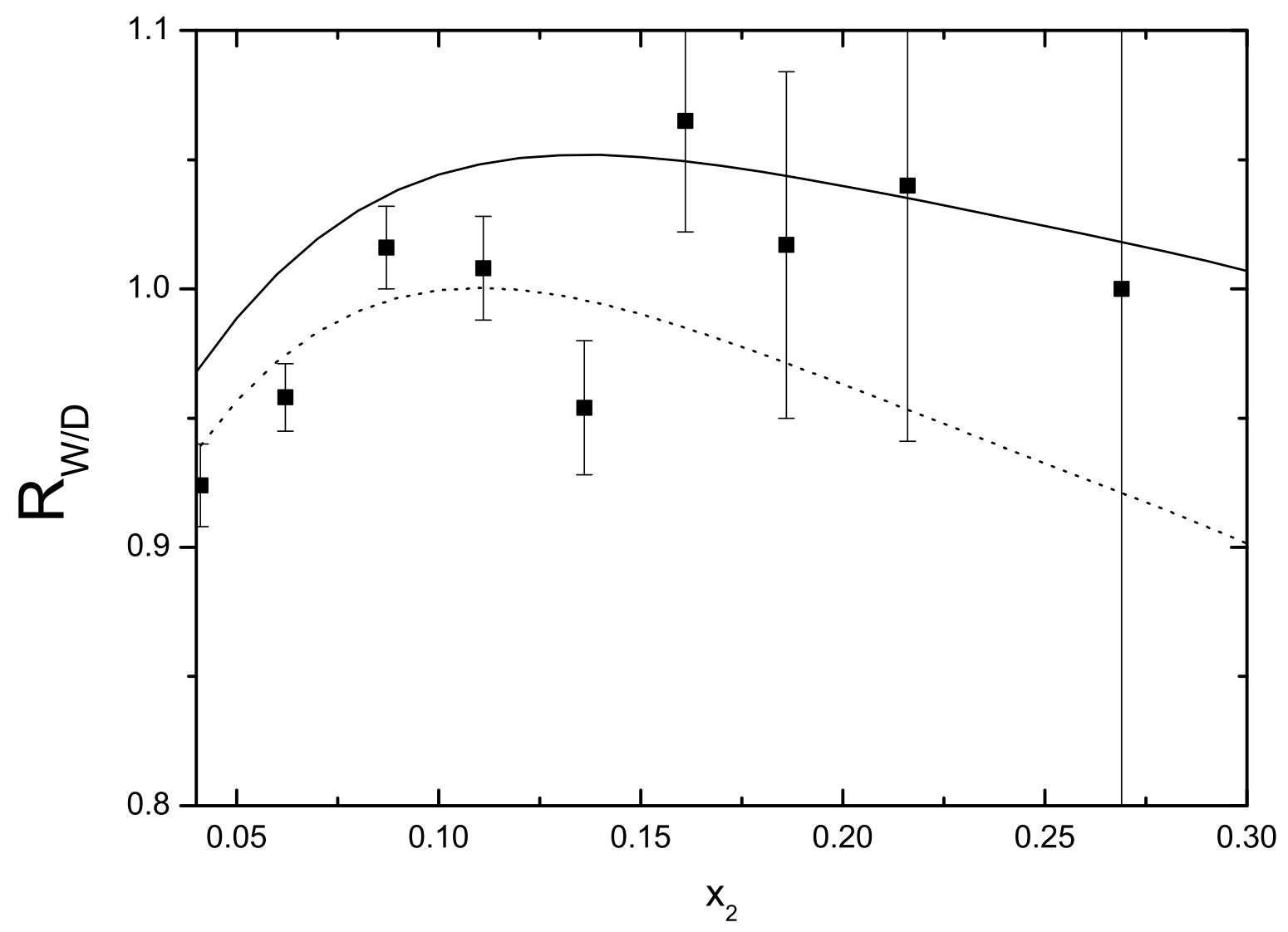

FIG. 2: The nuclear Drell-Yan cross section ratios $R_{W / D}\left(x_{2}\right)$ of tungsten versus deuterium. The comments are the same as Fig.1. 